\title{
Endoscopic 'hybrid' treatments for gastric varices: Do we need belt and suspenders?
}

\section{다 (1) $\odot$}

\author{
Author \\ Kenneth F. Binmoeller \\ Institution \\ Paul May and Frank Stein Interventional Endoscopy Center, \\ California Pacific Medical Center, San Francisco, California, \\ United States \\ Bibliography \\ DOI https://doi.org/10.1055/a-1024-3852 | \\ Endoscopy International Open 2020; 08: E228-E230 \\ (c) Georg Thieme Verlag KG Stuttgart · New York \\ eISSN 2196-9736
}

\author{
Corresponding author \\ Kenneth. F. Binmoeller, MD, Interventional Endoscopy \\ Services, California Pacific Medical Center, 2351 Clay St, \\ 6th Floor, San Francisco, CA 94115 \\ Fax: +1-415-600-1416 \\ BinmoeK@sutterhealth.org
}

Conceptually, intravascular injection of cyanoacrylate (CYA) glue should be the ideal treatment to stop bleeding and prevent rebleeding from gastric varices. Native CYA, a liquid with a consistency similar to water, is injected through a standard sclerotherapy needle into the varix lumen. Upon contact with a physiologic ionic medium like blood, the glue rapidly polymerizes, forming a rock-hard undegradable substance. The glue plugs up the varix lumen, resulting in rapid hemostasis (in the case of active bleeding) and prevention of rebleeding.

Interventional radiologists were the first to use the CYA glue bucrylate to treat bleeding esophago-gastric varices via a percutaneous transhepatic approach in 1978 [1]. Endoscopists quickly adopted the catheter-based approach, with the first report of bucrylate injection to treat esophageal varices in 1984 and gastric varices in 1986 [2,3]. In the decades to follow, numerous publications have validated the efficacy and relative safety of glue injection. A Cochrane review of three randomized controlled trials (RCTs) in 2015showed CYA injection of gastric varices to carry a significantly lower risk of rebleeding compared to endoscopic variceal ligation (EVL) [4]. Today, glue injection is practiced worldwide as first-line treatment for gastric varices, as recommended by the American Association for the Study of Liver Diseases and Baveno IV guidelines (Class I, Level B) $[5,6]$.

The impressive results of CYA therapy come at the trade-off of a potentially devastating complication: systemic glue embolization. Numerous case reports document embolization to virtually every part of the body, including the limbs, intestines, spleen, lungs, coronary arteries, and brain [7]. Routine thoracic computed tomography (CT) imaging after gastric variceal injection of glue ( $\mathrm{N}$-butyl-2-cyanoacrylate) diluted 1:1 with lipiodol revealed an alarmingly high rate of embolization to the lungs in nearly $60 \%$ of patients [8]. Although usually asymptomatic and inconsequential, clinical sequelae of embolization can be severe-and sometimes fatal. If embolization is survived, sepsis may follow when the embolus serves as a nidus of infection.

Ongoing developmental work has focused on strategies to mitigate risk of systemic CYA embolization. Modifications of injection technique have been proposed, including avoidance of overdilution with lipiodol in addition to aliquotting at most $1 \mathrm{~mL}$ of glue per syringe to restrain against "over-enthusiastic" injection [7]. In 2004, our group reported on use of 2-octyl-cyanoacrylate, a longer alkyl group CYA with a prolonged polymerization rate enabling a slower "drop-by-drop" delivery of the glue into the varix [9]. In 2007, Romero-Castro and colleagues in Seville described a small case series using EUS to targeting the perforating "feeder vessel" rather than the varix lumen proper under conventional endoscopic guidance [10]. The aim was to minimize the amount of CYA needed to achieve obliteration of GV and thereby potentially reduce the risk of embolization. In this series, a mixture of glue with lipiodol enabled fluoroscopic visualization of the injected vessel and confirmation that the feeder vessel had been accurately targeted. No recurrent bleeding or complications were observed during the follow-up period.

What alternative embolic agents may be capable of achieving similar results to CYA, but with a lower risk of embolization? The interventional radiologist's toolbox includes coils. Made of a metal alloy with attached synthetic fibers, coils have the advantage of a very low risk of migration when properly sized to the varix diameter. However, a large number of coils are needed to sufficiently occlude a large varix. In a case series of four patients who underwent endoscopic injection of coils as sole ther- 
apy to treat gastric variceal bleeding, up to 22 coils (0.035-inch, 50 to $150-\mathrm{mm}$ length) were required per patient, with a mean of nine coils [11]. The success rate was $75 \%$ (3 of 4 patients). In a retrospective study of 11 patients who underwent endoscopic ultrasound (EUS)-guided coil treatment of the perforating vein, a mean of 5.8 coils were deployed per patient [8]. The success rate of coiling alone (1-3 sessions) was $82 \%$, with two of 11 patients requiring additional CYA glue treatment. Interestingly, using transhepatic access to gastric varices, interventional radiologists have previously observed that use of coils as sole therapy resulted in high recanalization rates, attributed to the persistently elevated portal pressure [12].

How about a hybrid approach, combining coil and glue injection? Theoretically, initial deployment of an intravascular coil should slow flow in the varix and provide a scaffold for glue adherence, retaining the glue at the site of injection and potentially reducing the risk of glue embolization. Our group reported long-term results in a series of 152 patients with gastric varices with a mean follow-up of 436 days [13]. Therapeutic success was $99 \%$ with an overall rebleeding rate of $16 \%$, of which $8 \%$ was from gastric varices. Immediate post procedure embolization was not observed, but one patient developed pulmonary embolism 1 week after hospital discharge.

Another embolic agent in the interventional radiologist's toolbox is gelfoam. Made of purified pork skin gelatin, gelfoam has undergone very little evolution since its introduction in the 1940 s. The embolization effect is attributed to its physical property of absorbing up to 45 times its weight in fluid and its capacity to expand up to $200 \%$ in vivo [14]. Gelfoam is biodegradable (hence an "absorbable gelatin sponge") with liquefaction occurring in 7 days, and is therefore classified as a temporary embolic agent. This is in contrast to CYA, which is a permanent embolic agent. At first glance, gelfoam would thus not be a logical substitute for CYA for the treatment of gastric varices due to concerns for recanalization in the setting of persistent portal hypertension. A recent case series in the radiological literature may reflect the detriment of gelfoam's evanescent nature. After plug-assisted retrograde transvenous obliteration (PARTO) using gelfoam as the sole embolic agent for the treatment of gastric variceal hemorrhage, supplemental coiling is necessary to achieve durable obliteration in a subset of patients (15.8\% in one study of 18 treated patients) [14].

In this issue of Endoscopy International Open, Bazarbashi et al. from the Brigham and Women's Hospital report on a retrospective case series of EUS-guided coil injection followed by gelfoam for the treatment of bleeding gastric varices [15]. The authors have borrowed from a modification of the traditional method of balloon-occluded retrograde transvenous obliteration (BRTO) using a combination of coils and gelfoam (CARTO), described by Lee et al in a case series of 20 patients in 2014 [16]. Using an EUS-guided approach the authors similarly use this combination of embolization agents and have demonstrated technical feasibility and relative safety of this hybrid procedure. The technical success was reported to be $100 \%$ with no rebleeding or required reintervention during mean follow-up of 6 months. The authors concluded that the technique theore- tically minimizes embolic complications and the need for re-intervention.

Although technically "hybrid", it is important that the reader appreciate a critical distinction between the approach of combining coil and gelfoam described in the Boston study and that of the previously reported combination of coils and glue [13, $17,18]$. In the current study in question, prior to injecting gelfoam, coils were deployed to significantly reduce Doppler flow on EUS until an absence of contrast run-off on fluoroscopy was achieved. By contrast, the intent of coil deployment prior to glue injection is the entrapment of glue on the coil. Whereas only one coil is typically required prior to glue injection to fulfill the "scaffold" function, multiple coils (averaging 8 per case) were required to achieve the endpoint of absence of contrast run-off prior to gelfoam injection. The distinction is critical because varix obliteration may have been achieved with coils alone in the Boston study. What then is the true added value of gelfoam? The author's conclusion that gelfoam may be an "acceptable and possibly safer alternative to cyanoacrylate" is misleading because gelfoam did not substitute for CYA as it is currently used in combination with coiling. Whereas gelfoam was the added adjuvant treatment in the Boston study, coils are the adjuvant treatment (to retain the glue at the site of CYA injection) using the combined coil and glue approach. A similar use of coils as an adjuvant treatment prior to gelfoam injection would not make sense since gelfoam lacks adhesive properties. If a true "apples-to-apples" comparison of the two hybrid approaches were conducted, it is likely that risk of embolization using gelfoam would be higher.

Do we need suspenders added to the belt? Only RCTs can answer this question. For the hybrid coil and glue approach, we need to determine if adding coils (suspenders) prior to glue injection (belt) significantly reduces the risk of glue embolization. In a small pilot RCT of 32 patients, Lobo et al from Sao Paulo, Brazil obtained thoracic CT scans after treatment [19]. Asymptomatic pulmonary embolism occurred in 4 (25\%) patients treated with the hybrid coil and glue treatment and eight (50\%) in the glue alone patients. The difference did not reach statistical significance $(P=0.144)$, but this may represent a type 2 error due to underpowering. The results clearly justify a larger RCT. For the hybrid coil and gelfoam approach, we need to first determine if adding gelfoam (suspenders) to coils alone (belt) affords any clinical benefit. It would only be after proving a significant benefit to adding gelfoam to coils that a RCT comparing the two hybrid approaches should be performed. Pending the results of these studies, the best approach should be individualized based on operator experience and anecdotal outcomes. Having personally witnessed severe complications from glue embolization after EUS-guided glue injection, including hemiplegia in a patient with a patent foramen ovale, this author has instituted a hybrid approach of coiling prior to glue injection over the past decade. Experience will continue to remain anecdotal with low levels of evidence until well-designed, adequately powered RCTs are conducted. 
Competing interests

None

\section{References}

[1] Lunderquist A, Borjesson B, Owman T et al. Isobutyl 2-cyanoacrylate (bucrylate) in obliteration of gastric coronary vein and esophageal varices. AJR Am J Roentgenol 1978; 130: 1-6

[2] Gotlib JPZP. Une nouvelle technique de traitementendoscopique des varices oesophagiennes: I'obliteration. Endosc Dig 1984; 7: 2

[3] Soehendra N, Nam V, Grimm H. Endoscopic obliteration of large esophagogastric varices with bucrylate. Endoscopy 1986; 18: 25-26

[4] Rios Castellanos E, Seron P, Gisbert JP et al. Endoscopic injection of cyanoacrylate glue versus other endoscopic procedures for acute bleeding gastric varices in people with portal hypertension. Cochrane Database Syst Rev 2015; 5: 1-86

[5] Garcia-Tsao G, Abraldes JG, Berzigotti A et al. Portal hypertensive bleeding in cirrhosis: Risk stratification, diagnosis, and management: 2016 practice guidance by the American Association for the study of liver diseases. Hepatology 2017; 65: 310-335

[6] de Franchis R, Baveno VIF. Expanding consensus in portal hypertension: Report of the Baveno VI Consensus Workshop: Stratifying risk and individualizing care for portal hypertension. J Hepatol 2015; 63: 743-752

[7] Cameron R, Binmoeller KF. Cyanoacrylate applications in the Gl tract. Gastrointest Endosc 2013; 77: 846-857

[8] Romero-Castro R, Ellrichmann M, Ortiz-Moyano C et al. EUS-guided coil versus cyanoacrylate therapy for the treatment of gastric varices: a multicenter study (with videos). Gastrointest Endosc 2013; 78: 711-721

[9] Rengstorff D, Binmoeller K. A pilot study of 2-octyl cyanoacrylate injection for treatment of gastric fundal varices in humans. Gastointest Endosc 2004; 59: 553-558
[10] Romero-Castro R, Pellicer-Bautista F], Jimenez-Saenz M et al. EUSguided injection of cyanoacrylate in perforating feeding veins in gastric varices: results in 5 cases. Gastrointest Endosc 2007; 66: 402-407

[11] Romero-Castro R, Pellicer-Bautista F, Giovannini M et al. Endoscopic ultrasound (EUS)-guided coil embolization therapy in gastric varices. Endoscopy 2010; 42: E35-E36

[12] Coldwell DM, Stokes KR, Yakes WF. Embolotherapy: agents, clinical applications, and techniques. Radiographics 1994; 14: 623-643 quiz 645-646

[13] Bhat YM, Weilert F, Fredrick RT et al. EUS-guided treatment of gastric fundal varices with combined injection of coils and cyanoacrylate glue: a large U.S. experience over 6 years (with video). Gastrointest Endosc 2016; 83: 1164-1172

[14] Chang MY, Kim MD, Kim T et al. Plug-assisted retrograde transvenous obliteration for the treatment of gastric variceal hemorrhage. Korean | Radiol 2016; 17: 230-238

[15] Bazarbashi AN, Wang T], Thompson CT et al. Endoscopic ultrasoundguided treatment of gastric varices with coil embolization and absorbable hemostatic gelatin sponge: a novel alternative to cyanoacrylate. Endosc Int Open 2020; 08: E221-E227

[16] Lee EW, Saab S, Gomes AS et al. Coil-assisted retrograde transvenous obliteration (CARTO) for the treatment of portal hypertensive variceal bleeding: preliminary results. Clin Transl Gastroenterol 2014; 5: e61

[17] Binmoeller KF. Endoscopic ultrasound-guided coil and glue injection for gastric variceal bleeding. Gastroenterol Hepatol (NY) 2018; 14: 123-126

[18] Binmoeller KF, Weilert F, Shah JN et al. EUS-guided transesophageal treatment of gastric fundal varices with combined coiling and cyanoacrylate glue injection (with videos). Gastrointest Endosc 2011; 74: 1019-1025

[19] Lobo MRA, Chaves DM, DE Moura DTH et al. Safety and efficacy of eus-guided coil plus cyanoacrylate versus conventional cyanoacrylate technique in the treatment of gastric varices: a randomized controlled trial. Arq Gastroenterol 2019; 56: 99-105 